\title{
Efecto de diferentes láminas de riego sobre la producción y calidad de fresa (Fragaria sp.)
}

\section{Effect of different irrigation water levels on the production and quality of the strawberry (Fragaria sp.)}

YULI ALEXANDRA DEAQUIZ1

JAVIER GIOVANNI ÁLVAREZ-HERRERA' ${ }^{1,2}$

LIDA PAOLA PINZÓN-GÓMEZ1

\section{Fresa variedad Ventana en fructificación.}

Foto: Y.A. Deaquiz

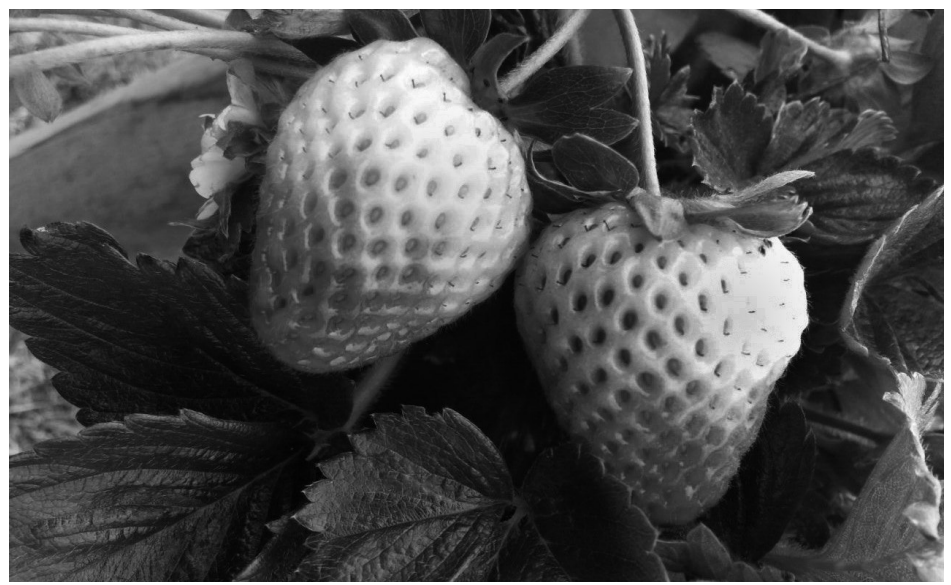

\section{RESUMEN}

La fresa es un cultivo de gran importancia económica para muchos agricultores de Colombia, principalmente para los de clima de frío de Boyacá y Cundinamarca; sin embargo, en estas zonas el bajo nivel de tecnificación del sistema de producción y el desconocimiento del manejo adecuado del riego en el cultivo de fresa conlleva al uso ineficiente del recurso hídrico. Por lo anterior, el objetivo de esta investigación fue evaluar el efecto de diferentes láminas de riego sobre la producción y calidad poscosecha de los frutos de fresa var. Ventana. Se utilizó un diseño experimental completamente al azar con cinco tratamientos y cuatro repeticiones. Los tratamientos correspondieron a cinco láminas de riego determinadas por cinco coeficientes multiplicadores de la evaporación $(0,8 ; 1,0 ; 1,2 ; 1,4$ o 1,6 de la evaporación medida dentro del invernadero). Para la siembra se utilizó turba rubia como sustrato, las aplicaciones se realizaron mediante sistema de riego por goteo. La lámina de riego de 1,6 presentó los mejores resultados de masa fresca de hojas, masa seca de hojas, corona y peciolos, área foliar y sólidos solubles totales de frutos; mientras que la lámina de riego de 1,4 obtuvo los mejores resultados de masa fresca de corona y peciolos, raíz, masa seca de raíz, frutos y relación de madurez. El coeficiente de riego de 1,4 presentó los mejores rendimientos y mantuvo una alta eficiencia en el uso del agua. El manejo de diferentes láminas de riego afecta las características organolépticas de los frutos de fresa.

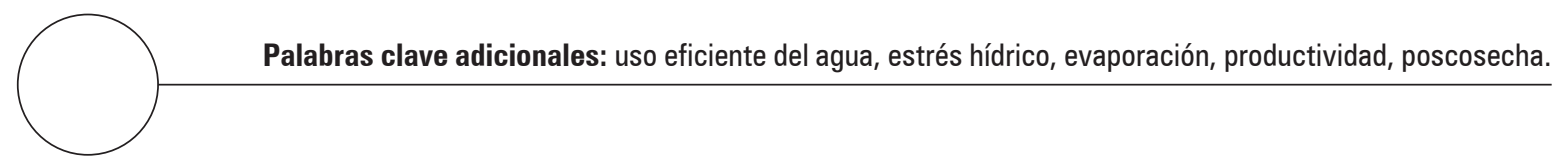

\footnotetext{
Facultad de Ciencias Agropecuarias, Grupo de Investigaciones Agrícolas, Universidad Pedagógica y Tecnológica de Colombia, Tunja (Colombia).

2 Autor para correspondencia: jgalvarezh@gmail.com
} 


\section{ABSTRACT}

The strawberry is a crop of great economic importance for many farmers in Colombia, mainly in the cold climatic zones of Boyacá and Cundinamarca; however, in these areas, the low level of technology in the production system and the lack of proper irrigation management in the strawberry cultivation lead to an inefficient use of water resources. Therefore, the objective of this research was to evaluate the effect of different water levels on the production and postharvest quality of strawberry fruits, var. Ventana. A completely randomized design with five treatments and four replications was used. The treatments consisted of five water levels as determined by five evaporation multiplication coefficients $(0.8,1.0,1.2$, 1.4 or 1.6 times the evaporation measured inside the greenhouse). Peat moss was used as a substrate for the planting; the water applications were carried out using a drip irrigation system. The irrigation coefficient of 1.6 provided the best results for the leaf fresh mass, dry mass of the leaves, crown and petioles, leaf area and total soluble solids of the fruit; while, the irrigation level of 1.4 resulted in the highest fresh mass of the crown and petioles, and roots, root dry mass, fruit and maturity index. The irrigation coefficient of 1.4 presented the best yield and maintained a high water efficiency. The management of different levels of water affects the organoleptic properties of strawberry fruits.

Additional key words: water use efficiency, water stress, evaporation, productivity, postharvest.

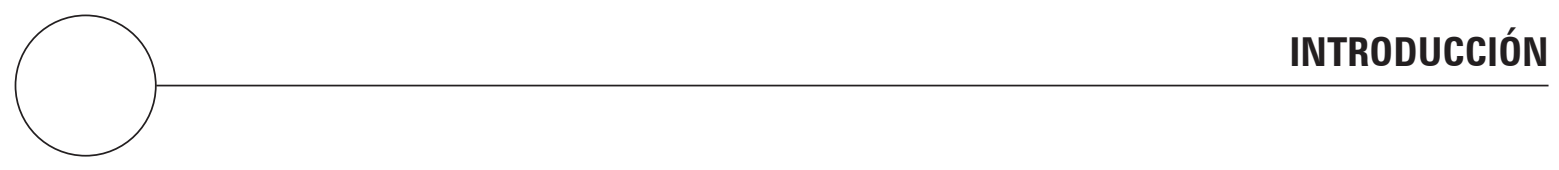

La fresa (Fragaria sp.) es un cultivo de gran importancia económica para muchos agricultores del país, principalmente para los de clima frío de Boyacá y Cundinamarca. Este cultivo tiene una demanda de 1.254,6 $\mathrm{mm}$ de agua durante su ciclo, pero el agricultor generalmente abusa de este recurso aplicando cantidades superiores a las requeridas por la planta, ya sea por desconocimiento o por considerar que con una mayor cantidad de agua se obtienen mayores rendimientos (Vázquez-Gálvez et al., 2008). El riego se debe realizar de forma óptima en el cultivo de fresa debido a que este cultivo es sensible al déficit hídrico (Hanson y Bendixen, 2004), viéndose afectado fuertemente el crecimiento de las plantas y la producción (Krüger et al., 1999), además, el estado hídrico de la planta tiene acción directa sobre procesos metabólicos y fisiológicos, dando como resultado una mayor resistencia estomática (Pires et al., 2006).
La apertura de los estomas es uno de los principales procesos fisiológicos afectados bajo condiciones de estrés hídrico (Taiz y Zeiger, 2006), y es muy importante porque es el principal control del paso del agua durante los intercambios gaseosos. Por otra parte, las condiciones de exceso de agua también son perjudiciales para el crecimiento de la fresa, el estado fitosanitario y el rendimiento (Kirnak et al., 2003). En este contexto, los estudios que dan apoyo al uso racional del agua en distintas condiciones ambientales deben ser de gran importancia (Hanson y Bendixen, 2004).

León et al. (2003) indican que la fresa requiere alta humedad para su establecimiento y así lograr plantas bien desarrolladas, de igual manera, en la etapa de maduración y cosecha, estas plantas presentan altas necesidades hídricas, además, lo más conveniente es aplicar pequeñas 
dosis con alta frecuencia, debido a que el sistema radical es superficial, por lo que recomiendan una frecuencia de riego de $2 \mathrm{~d}$, no obstante, los productores prefieren aplicar riego en exceso para evitar periodos de deficiencia hídrica, pero la alta humedad puede ocasionar lixiviación de nutrientes (Ojeda-Real et al., 2008), así como también un aumento en la incidencia de enfermedades (Merchán-Gaitán et al., 2014).

Mediante la regulación del riego se logra un balance entre el crecimiento vegetativo y reproductivo, ya que un exceso de vigor en las plantas tiene efectos negativos sobre la composición química de algunos frutos, como en el caso de la vid (Dry et al., 2001). Además, con el tiempo, el agua ha adquirido mayor importancia ya que es un recurso limitado y no siempre disponible (GWP, 2000) y actualmente ya se han aumentado las restricciones para el uso de este líquido (Leskovar y Pccini, 2005). Según Ryu et al. (2014), la programación del riego puede ahorrar alrededor de un $50 \%$ de agua y el riego por goteo podría influir favorablemente en la altura de la planta, el índice de área foliar, el peso del fruto y la calidad en un 10-15\%. Así, en los últimos años la investigación sobre la eficiencia en el uso del agua ha aumentado y se ha elevado la inversión en investigación con el fin de desarrollar planes y sistemas de riego que hagan la producción de alimentos y el manejo del recurso agua más sostenible (Cosgrove y Rijisberman, 2014).

Por lo anterior, el objetivo de esta investigación fue evaluar el efecto de diferentes láminas de riego sobre la producción y calidad poscosecha de fresa, como una alternativa viable para aumentar la producción y la calidad de los frutos y que a la vez garanticen un uso eficiente del agua.

\section{MATERIALES Y MÉTODOS}

El experimento se realizó en un invernadero de plástico en la ciudad de Tunja (Boyacá), que se encuentra a una altitud de $2.690 \mathrm{msnm}$ y tiene las siguientes coordenadas: longitud $73^{\circ} 23^{\prime}$ oeste, latitud $5^{\circ} 32^{\prime}$ norte. La temperatura media dentro de la cubierta plástica fue de $19^{\circ} \mathrm{C}$ y la humedad relativa del $75 \%$.

Se utilizó un diseño experimental completamente al azar con cinco tratamientos y cuatro repeticiones. Los tratamientos correspondieron a cinco láminas de riego determinadas por cinco coeficientes multiplicadores de la evaporación $(0,8 ; 1,0 ; 1,2 ; 1,4$ o 1,6 de la evaporación medida dentro del invernadero), para un total de 20 unidades experimentales (UE). Cada UE estuvo compuesta por tres plantas. Los coeficientes utilizados fueron escogidos de acuerdo con los requerimientos hídricos del cultivo de fresa. Se utilizó turba rubia como sustrato, ya que comercialmente es el más utilizado y para darle un aporte nutricional los primeros días del establecimiento del cultivo. Las plantas de fresa de variedad Ventana fueron sembradas en materas plásticas con capacidad para $4 \mathrm{~L}$ de sustrato. Durante la etapa de floración, se aplicó un fertilizante foliar (10 N - 30 P - $10 \mathrm{~K})$ con aminoácidos y nutrientes complementarios: magnesio $(\mathrm{Mg})$, boro $(\mathrm{B})$, zinc $(\mathrm{Zn})$ y cobre $(\mathrm{Cu})$. Los tratamientos de riego se iniciaron $15 \mathrm{~d}$ después del trasplante (ddt) finalizaron con la cosecha de los frutos. Las aplicaciones se realizaron mediante sistema de riego por goteo.

La lámina de riego se aplicó de acuerdo con los coeficientes establecidos en los tratamientos, para lo cual se midió diariamente la evaporación en un tanque evaporímetro construido a escala del tanque clase A (diámetro de $29 \mathrm{~cm}$ y altura de $6,1 \mathrm{~cm}$ ) con el fin de poder establecer la cantidad de agua a aplicar y el consumo de agua durante todo el cultivo. Para determinar las dosis de agua a aplicar se utilizó la siguiente ecuación:

$$
\text { Lámina }=\frac{E t p * C * A}{\eta_{\mathrm{r}}}
$$

En donde, Etp = evapotranspiración en $\mathrm{mm}$ medida en el tanque evaporímetro; $\mathrm{C}=$ coeficien- 
te multiplicador; $\mathrm{A}=$ área de la matera $\left(\mathrm{cm}^{2}\right)$; $\eta_{\mathrm{r}}=$ eficiencia del riego $(0,9)$. Las variables de respuesta determinadas (al finalizar la cosecha) fueron masa fresca y seca de raíz, corona y peciolos, hojas (incluyendo las descartadas en los deshojes realizados) y frutos, para lo cual, se tomaron las plantas, se dividieron en los respectivos órganos y se secaron en una mufla a $70^{\circ} \mathrm{C}$ durante 48 horas, luego se pesaron en una balanza electrónica de precisión 0,001. El área foliar se evaluó con un medidor portátil de área foliar CI-202 (Seedmech-Wintersteiger, Ried, Austria). La eficiencia en el uso del agua se determinó al dividir la masa seca de hojas en el agua total aplicada en cada tratamiento.

Para determinar los datos de las variables de calidad, se realizó recolección manual (tres cosechas). Los frutos se cosecharon según la Norma Técnica Colombiana para Fragaria NTC 4103 (Icontec, 1996) y se les midieron sus características fisicoquímicas. Se determinó la acidez total titulable (ATT) calculada como porcentaje de ácido cítri$\mathrm{co}$, utilizando una mezcla de $5 \mathrm{~g}$ de jugo de frutos y agua destilada a la cual se le agrego 3 gotas de fenoftaleína, luego se realizó la titulación con hidróxido de sodio $(\mathrm{NaOH})$ y la valoración se basó en el cambio de color como indicador, el porcentaje de acidez se halló con la siguiente fórmula:

$$
\% \text { Acidez }=(A * B * C) * 100 / D
$$

En donde: $\mathrm{A}=$ volumen de $\mathrm{NaOH}$ gastado; $\mathrm{B}=$ normalidad del $\mathrm{NaOH}(0,097) ; \mathrm{C}=$ peso equivalente expresado en g de ácido predominante en el fruto (ácido cítrico 0,064 $\mathrm{g} \mathrm{meq}^{-1}$ ); D = peso en gramos de la muestra utilizada (5 g).

La concentración de sólidos solubles totales (SST) se halló a través de mediciones de ${ }^{\circ}$ Brix con un refractómetro digital marca Hanna HI 968033 (Hanna Instruments, Eibar, España) de rango 0 a $85 \%$ con precisión $0,1^{\circ}$ Brix. La relación de madurez (RM) se halló mediante la relación SST/ATT.
Los datos fueron sometidos a pruebas de normalidad, posteriormente se realizó un análisis de varianza y luego una prueba de comparación de promedios de Tukey al 5\%, para cada uno de los tratamientos evaluados. Los análisis se realizaron con el software estadístico SAS® v. 8.1e (SAS Institute Inc., Cary, NC).

\section{RESULTADOS Y DISCUSIÓN}

\section{Masa fresca de órganos}

Se presentaron diferencias estadísticas significativas $(P \leq 0,05)$ en la masa fresca de hojas, corona y peciolos, raíz y frutos. Para el caso de la masa fresca de hojas, las plantas con mejor resultado (mayor peso) fueron las de la aplicación de un coeficiente de riego de 1,6 con un peso de 23,11 g, para corona y peciolos, el mejor coeficiente fue el de 1,4 , pero este no presentó diferencias con el coeficiente de 1,6 (figura 1A y 1B). En cuanto a la masa de raíz, el coeficiente de 1,0 presentó los mayores valores (figura 1C), concordando con los resultados obtenidos por Álvarez-Herrera et al. (2010), en romero.

La masa fresca de frutos no presentó diferencias significativas, no obstante el mejor resultado se obtuvo con el coeficiente de 1,4 (figura 1D); sin embargo, según (Silber y Bar-Tal, 2008), los frutos tras la aplicación de mayores cantidades de agua se favorecen, ya que hay mayor disponibilidad de nutrientes y los procesos de asimilación y translocación de asimilados hacia los frutos se realizan de forma más eficiente aumentando la masa fresca de estos.

Por otra parte, la cantidad de agua en los frutos depende de otros factores como el estatus hídrico de estos, Kader (2013) menciona que la cantidad de agua es directamente proporcional al déficit de presión de vapor entre el órgano y el ambiente, por lo que a mayor déficit de presión de vapor, menor cantidad de agua y así menor masa fresca. 

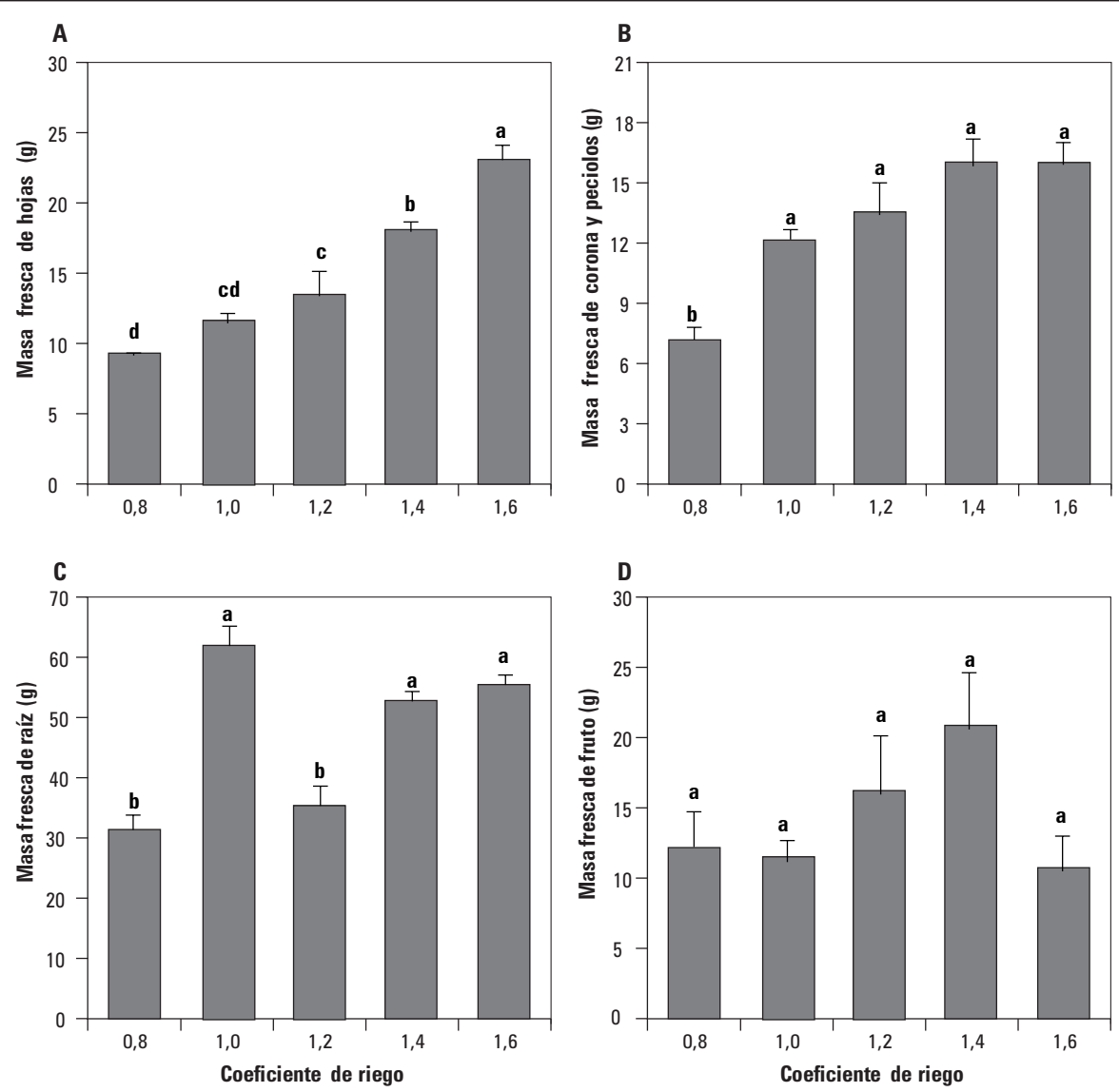

Figura 1. Efecto de la aplicación de diferentes láminas de riego en la masa fresca de A. Hojas, B. Corona y peciolos, C. Raíz y D. Frutos de plantas de fresa. Promedios con letras distintas indican diferencia significativa según la prueba de Tukey $(P \leq 0,05)$. Las barras de error indican el error estándar.

Con cada lámina de riego aplicada se obtuvieron diferentes valores de masa fresca para cada órgano, esto debido a que, según Galván-Tovar et al. (2003), la acumulación de masa fresca de corona y peciolos, y en especial la de hojas es más sensible al déficit hídrico que la de la raíz. Igualmente, mayores aplicaciones de agua hacen más eficiente el proceso de transporte de nutrientes a través del xilema y el floema, de esta manera las hojas, corona y peciolos reciben más agua y nutrientes, por otra parte, la existencia de diferencias en la masa fresca de raíces y en la parte aérea (corona, peciolos y hojas) se presenta por el ajuste osmótico efectuado, que permite regular las pérdidas en el follaje (Balaguera et al., 2008) aumentando la acumulación de biomasa fresca.
Los resultados presentados en los tratamientos reflejan que el suministro de diferentes cantidades de agua conlleva a una variación en la división celular y en la absorción de oxígeno, que son esenciales en los procesos metabólicos primarios de la planta como la fotosíntesis y la respiración, indispensables en el crecimiento y desarrollo de la planta lo que contribuye a incrementar la masa fresca (Taiz y Zeiger, 2006). Balaguera et al. (2008) mencionan que el aumento de absorción de agua y nutrientes promueven la síntesis de compuestos fotoasimilados en la parte aérea, razón por la cual, es probable que se registre el incremento de masa fresca en las estructuras de la planta. Igualmente, suministrar diferentes láminas de riego puede activar 
o incrementar los mecanismos de resistencia de las plantas a situaciones de sequía, induciendo la formación de raíces (Ramírez-Palma et al., 2005) más fibrosas y activas aumentando así el potencial de estas para una mayor absorción de nutrientes, reflejándose esto en la acumulación de biomasa en los diferentes órganos de la planta (Kumar y Dey, 2011).

\section{Masa seca de órganos}

El efecto de las láminas de riego influyó en la acumulación de masa seca de hojas, corona y peciolos, raíz y frutos, ya que se presentaron diferencias significativas entre tratamientos. El coeficiente de riego de 1,6 presentó los mejores resultados en masa seca de hojas y corona y pe- ciolos, con 7,0 y 7,8 g, respectivamente, mientras que el coeficiente de 1,0 y 0,8 obtuvo los menores valores en hojas (figura $2 \mathrm{~A}$ ) y corona y peciolos (figura $2 \mathrm{~B}$ ). Los resultados encontrados pueden deberse a que con una mayor lámina de riego aplicada las hojas y corona y peciolos reciben más agua y nutrientes, aumentando su crecimiento y por ende presentando mayor reserva de fotoasimilados (Álvarez-Herrera et al., 2010).

En cuanto a la masa seca de raíz y frutos, el coeficiente de 1,4 alcanzó la mayor masa seca con 12,95 y $2,19 \mathrm{~g}$, mientras que con la lámina de 0,8 se obtuvieron los menores valores de masa fresca de raíz (figura $2 \mathrm{C}$ y $2 \mathrm{D}$ ). Probablemente estos resultados debidos a que una menor difusión de oxígeno en la zona radicular causada por la falta de agua disminuye el

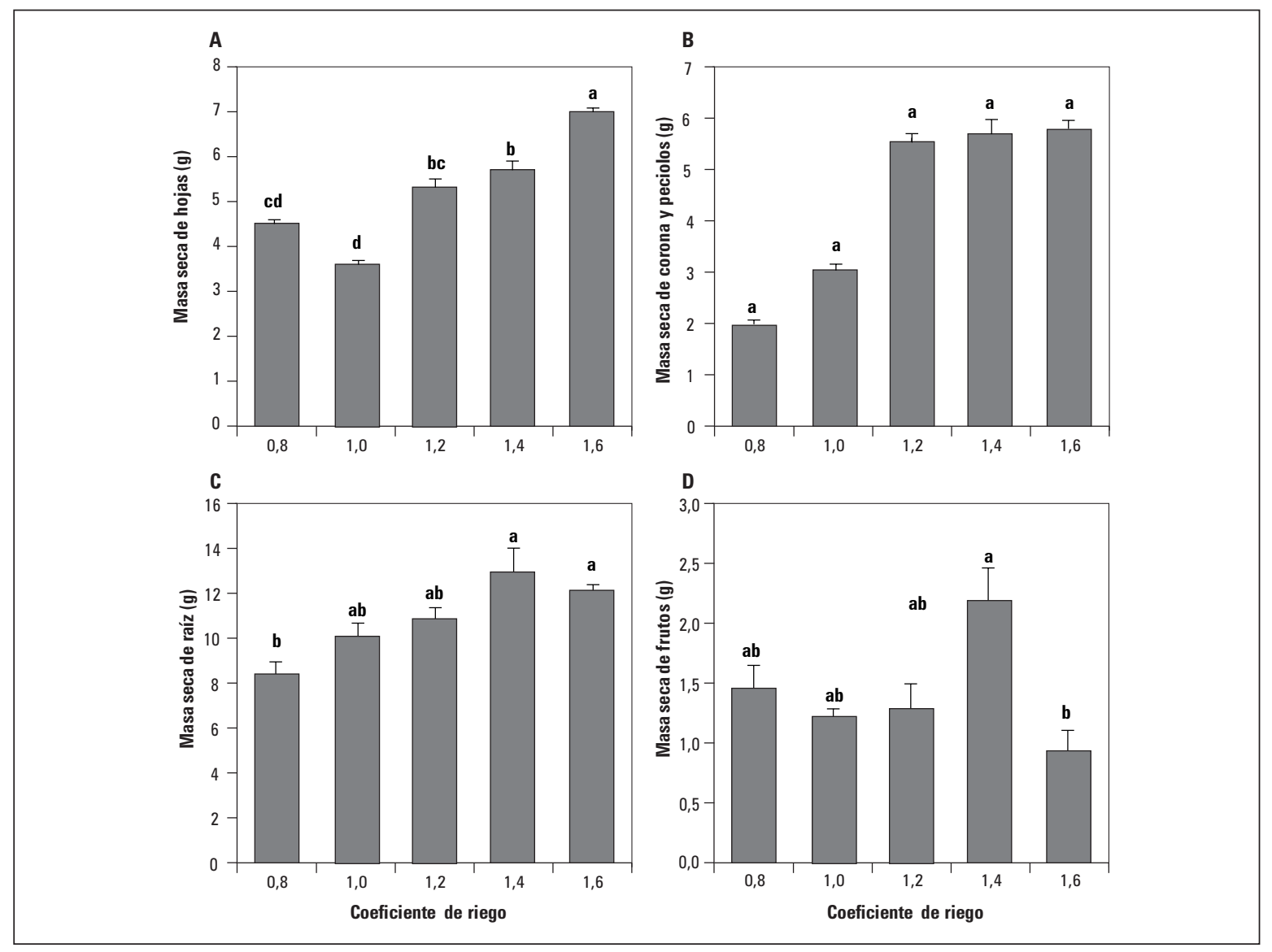

Figura 2. Efecto de la aplicación de diferentes láminas de riego en la masa seca de A. Hojas, B. Corona y peciolos, C. Raíz y D. Frutos de plantas de fresa. Promedios con letras distintas indican diferencia significativa según la prueba de Tukey $(P \leq 0,05)$. Las barras de error indican el error estándar. 
crecimiento de la raíz (Álvarez-Herrera et al., 2008). Por otra parte, el bajo contenido de agua disminuye los procesos de fotosíntesis, además de que el transporte de nutrientes minerales es menos eficiente a través de los haces vasculares de la planta (Prieto et al., 2010).

De acuerdo con lo anterior, se resalta la importancia del adecuado manejo del agua, ya que es uno de los factores que más limita la producción de biomasa en la planta, pues se ha comprobado que existe una relación directa entre la masa fresca y la masa seca, debido a que una mayor disponibilidad de agua influye directamente en la masa seca de la planta (Tadeo, 2000). La producción de materia seca en la parte aérea de la planta está altamente relacionada con la intercepción de la radiación fotosintéticamente activa, donde a medida que disminuye la disponibilidad de agua en el suelo se incrementa la traslocación de carbohidratos a la raíz (Núñez et al., 2005). Así mismo, excesos de agua estresan a las raíces, por lo que hay mayor síntesis de etileno y menor suministro de fitorreguladores como citoquininas y giberelinas al tallo disminuyendo el crecimiento longitudinal del tallo y la acumulación de biomasa (Baracaldo et al., 2014), afectando la adsorción y transporte de nutrientes y así la producción.

Por otro lado, las modificaciones en los patrones de acumulación de materia seca en los diferentes órganos se pueden explicar mediante la alteración en la tasa fotosintética, dado que en plantas de fresa expuestas a encharcamiento, se produce un rápido cierre de los estomas (Casierra-Posada y Vargas, 2007); así también, la variación en la masa seca del fruto, se podría explicar en la relación que este tiene con la masa seca de raíces, ya que ante la existencia de mayor cantidad de raíces, se incrementa la capacidad para obtener una absorción más eficiente de nutrientes y agua, aumentando la disponibilidad de asimilados para el desarrollo de los frutos (Viloria et al., 1998). Porro et al.
(2010) reportan que en uva una menor cantidad de agua aplicada reduce el tamaño de los frutos pero no la masa seca de los mismos.

\section{Área foliar}

Esta variable presentó diferencias estadísticas significativas entre las láminas de riego, en donde las plantas que fueron sometidas a la mayor aplicación de agua, presentaron un mayor desarrollo y mostraron los valores más altos de área foliar, así la mejor respuesta se obtuvo con el coeficiente de riego de 1,6 con 958,72 $\mathrm{cm}^{2}$ (figura 3). Lo anterior es similar a lo ocurrido en investigaciones de café con aplicación de distintos niveles de riego, en las cuales Casierra-Posada et al. (2009) encontraron que bajas láminas de riego generan reducción del área foliar, lo que disminuye la transpiración con el fin de evitar la deshidratación de las hojas. También se obtuvo una disminución en el crecimiento de las hojas en plantas de girasol sometidas a riego limitado (Cellier et al., 1998). Además, coincide con lo encontrado por Núñez et al. (2005) en plantas de fríjol, quienes obtuvieron reducción en el área foliar y disminución de número de hojas, cuando las plantas se desarrollaron bajo condiciones de déficit hídrico.

Al respecto, las plantas con mayor área foliar y ambiente favorable son capaces de utilizar mejor la energía solar siendo más eficientes en el proceso de fotosíntesis (Jarma et al., 1999). Por otra parte, como consecuencia del déficit hídrico, uno de los primeros efectos que se manifiestan en las hojas a nivel ecofisiológico es la reducción de la apertura estomática afectando la fotosíntesis y evitando la evapotranspiración (Chaves et al., 2010; Ibáñez et al., 2011), además se disminuye la turgencia y la expansión celular, por tal razón el crecimiento de las hojas se reduce afectando el área foliar. Igualmente el bajo contenido de agua no solo limita el tamaño sino también el número de hojas originado por una disminución en la tasa de crecimiento del tallo (Taiz y Zeiger, 2006). 


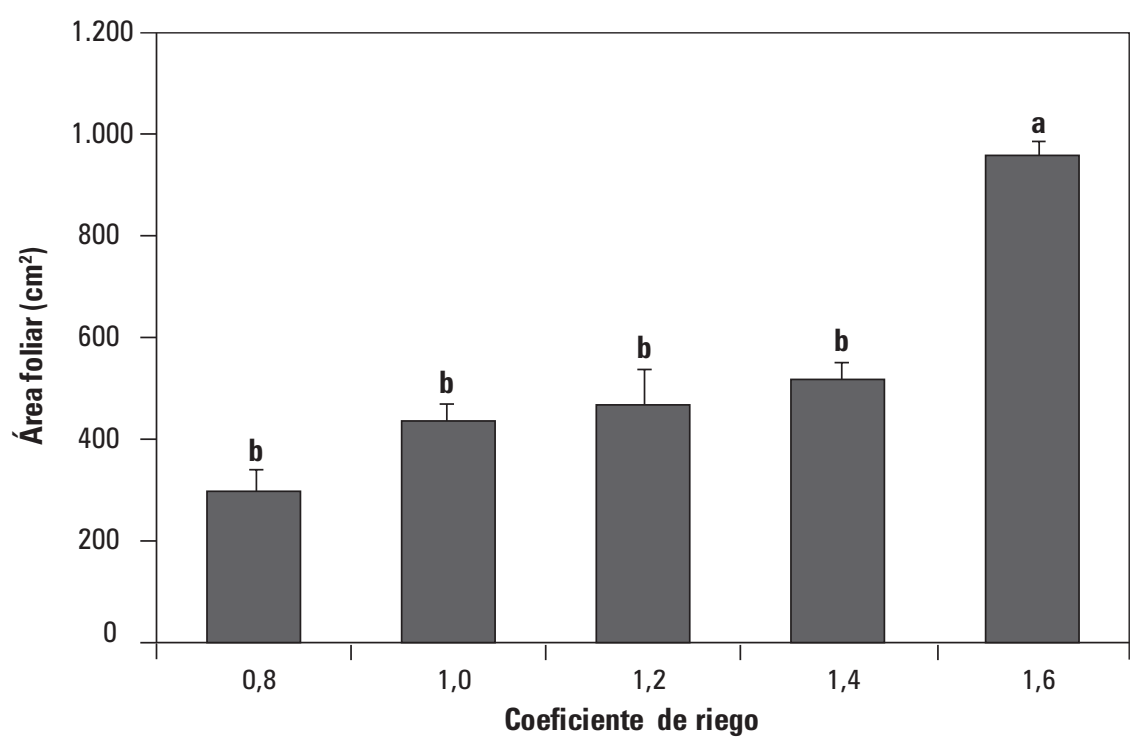

Figura 3. Área foliar de plantas de fresa sometidas a diferentes láminas de riego. Promedios con letras distintas indican diferencia significativa según la prueba de Tukey $(P \leq 0,05)$. Las barras de error indican el error estándar.

Sólidos solubles totales (SST) de los frutos

En esta variable se presentaron diferencias estadísticas significativas entre los tratamientos. Los frutos provenientes de plantas regadas con un coeficiente de 1,6 presentaron los valores más altos de SST con 7,58 ${ }^{\circ}$ Brix, seguido del tratamiento que fue regado con una lámina de 1,4 con $7,11^{\circ}$ Brix, por otra parte los coeficientes de 0,8 y 1,0 no presentaron diferencias significativas y el coeficiente de 1,2 registró los valores más bajos con $5,52{ }^{\circ}$ Brix (figura 4), re-

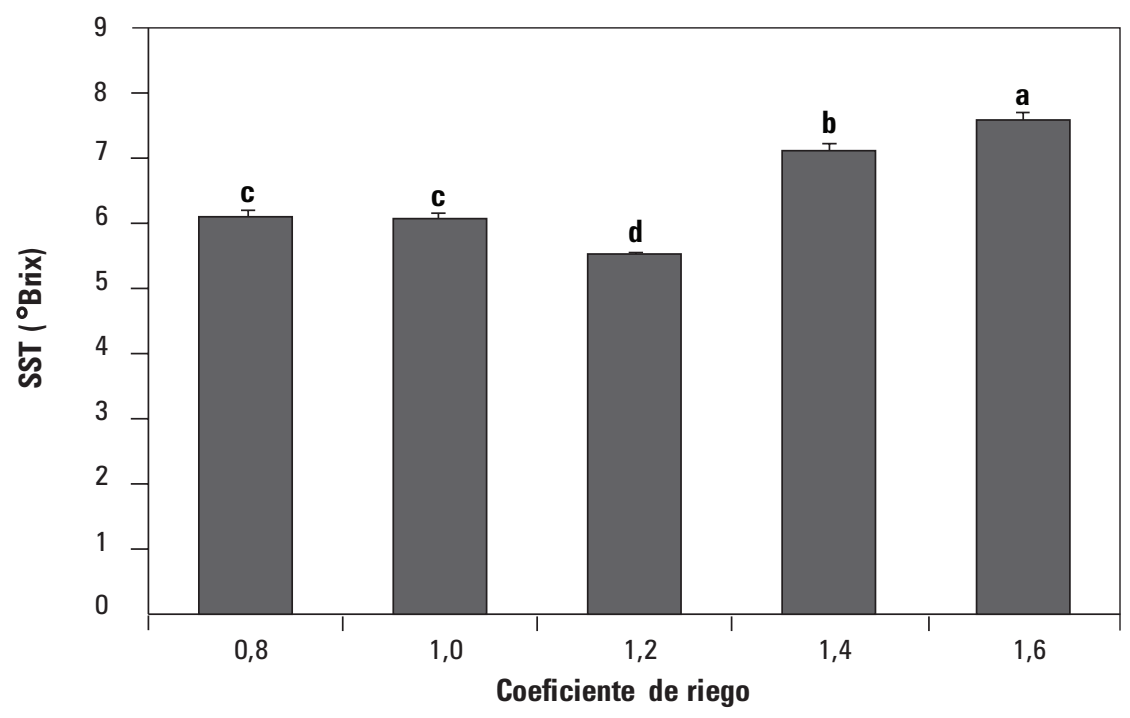

Figura 4. Sólidos solubles totales (SST) de frutos de fresa con aplicación de diferentes láminas de riego. Promedios con letras distintas indican diferencia significativa según la prueba de Tukey $(P \leq 0,05)$. Las barras de error indican el error estándar. 
sultados similares a los encontrados por Zeng et al. (2009) en melón, donde los valores de SST disminuyeron a medida que disminuyó la lámina de riego aplicada. Contrario a lo anterior se ha reportado un aumento de los SST debido a la disminución de la lámina de riego en frutos como manzana (Parés et al., 2010), durazno (Rufat et al., 2010), vid (Satta et al., 2011) y tomate (Beckles, 2012; Wang et al., 2011).

En contraste con lo encontrado en el presente estudio, Álvarez-Herrera et al. (2011) afirman que la aplicación de una mayor cantidad de agua disminuye la cantidad de ${ }^{\circ}$ Brix, debido a que la concentración de los azúcares se ve diluida por el mayor contenido de agua que presentan los frutos. Posiblemente el aumento de los SST en fresa pudo deberse a que se ha demostrado que existe un crecimiento paulatino de la concentración de los sólidos solubles totales (monosacáridos) durante la maduración, como consecuencia de la hidrólisis de los polisacáridos (homopolisacárido) (Ademir et al., 2002). Igualmente, los SST son un parámetro de calidad del fruto que depende de factores como la maduración, el cultivar, la nutrición de la planta y el estrés hídrico (Urrestarazu, 2004).

\section{Acidez total titulable (ATT) de los frutos}

Se presentaron diferencias estadísticas significativas entre los tratamientos, en donde los promedios más altos de ATT fueron obtenidos en los frutos regados con un coeficiente de 0,8 con $3,97 \%$, mientras que los coeficientes de 1,2 y 1,6 no presentaron diferencias significativas, $y$ a su vez las aplicaciones de agua de 1,0 y 1,4 de la evaporación presentaron los valores más bajos con 3,14\% y $3,05 \%$, respectivamente (figura 5), lo que permite afirmar que la lámina de riego influyó en el contenido de ATT en los frutos de fresa. Según Marschner (2002) con un déficit de agua, los frutos probablemente presentan una mayor respiración, situación que origina la conversión de ácidos a azúcares de forma más rápida. En tomate algunos autores (Patané et al., 2011; Favati et al., 2009; Marouelli y Silva, 2007) reportan que cuando las plantas de tomate sufren de estrés hídrico tienen una reducida absorción de agua, e incrementan la producción

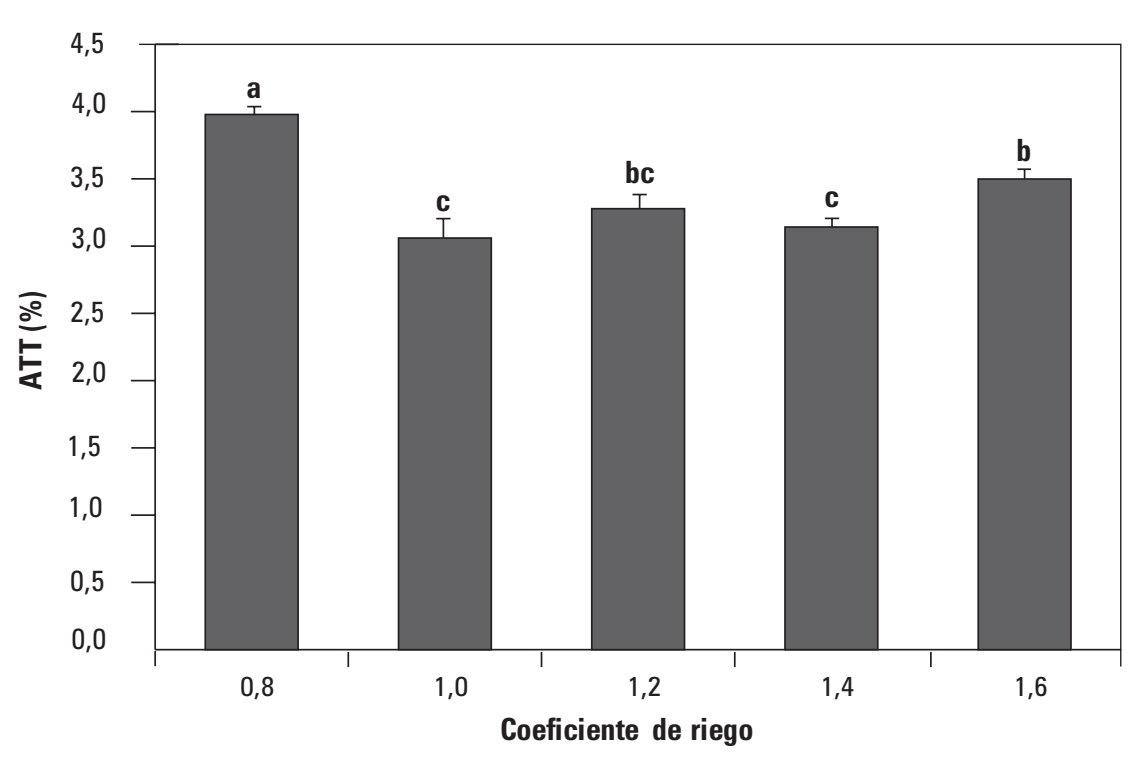

Figura 5. Acidez total titulable (ATT) de frutos de fresa con aplicación de diferentes láminas de riego. Promedios con letras distintas indican diferencia significativa según la prueba de Tukey $(P \leq 0,05)$. Las barras de error indican el error estándar. 
de ácidos en los frutos como mecanismo de adaptación al estrés.

Además, los ácidos generalmente disminuyen durante la maduración, ya que ellos son sustratos respiratorios o son convertidos a azúcares, que pueden ser considerados una fuente de energía y se esperaría que disminuyeran durante la actividad metabólica que se desarrolla en la maduración (Heredia et al., 1997), pues los ácidos orgánicos son después de los carbohidratos, el depósito energético más importante para el fruto (Osterloh et al., 1996).

\section{Relación de madurez de frutos}

En esta variable hubo diferencias estadísticas significativas entre las láminas de riego, donde el coeficiente de 1,4 presentó la mayor relación con 2,41; por su parte los coeficientes de 0,8 y 1,0 no presentaron diferencias significativas, mientras que los de 1,6 y 1,2 obtuvieron los valores más bajos (figura 6), lo cual se puede explicar debido al comportamiento de la ATT, ya que esta fue mayor con las menores aplicacio- nes de agua, mientras que los SST de los frutos de fresa disminuyeron por las bajas láminas de agua.

Lo anterior se debe a que los valores de ATT disminuyen progresivamente en función de la maduración del fruto, comportamiento inverso al de los SST, ya que los ácidos orgánicos disminuyen por procesos de hidrólisis durante la maduración (Paliyath y Murr, 2008). Además se observa que a medida que el fruto es más suculento o su contenido de agua es mayor esta relación se hace proporcional. Al respecto, Osterloh et al. (1996) afirman la importancia de la relación entre SST y ATT en el sabor del fruto y del jugo, teniendo en cuenta que cuando el fruto tiene un contenido alto de azúcares, el nivel de los ácidos debe ser suficientemente elevado para satisfacer el gusto del consumidor.

\section{Uso eficiente del agua}

En esta variable se presentaron diferencias estadísticas significativas entre las láminas de rie-

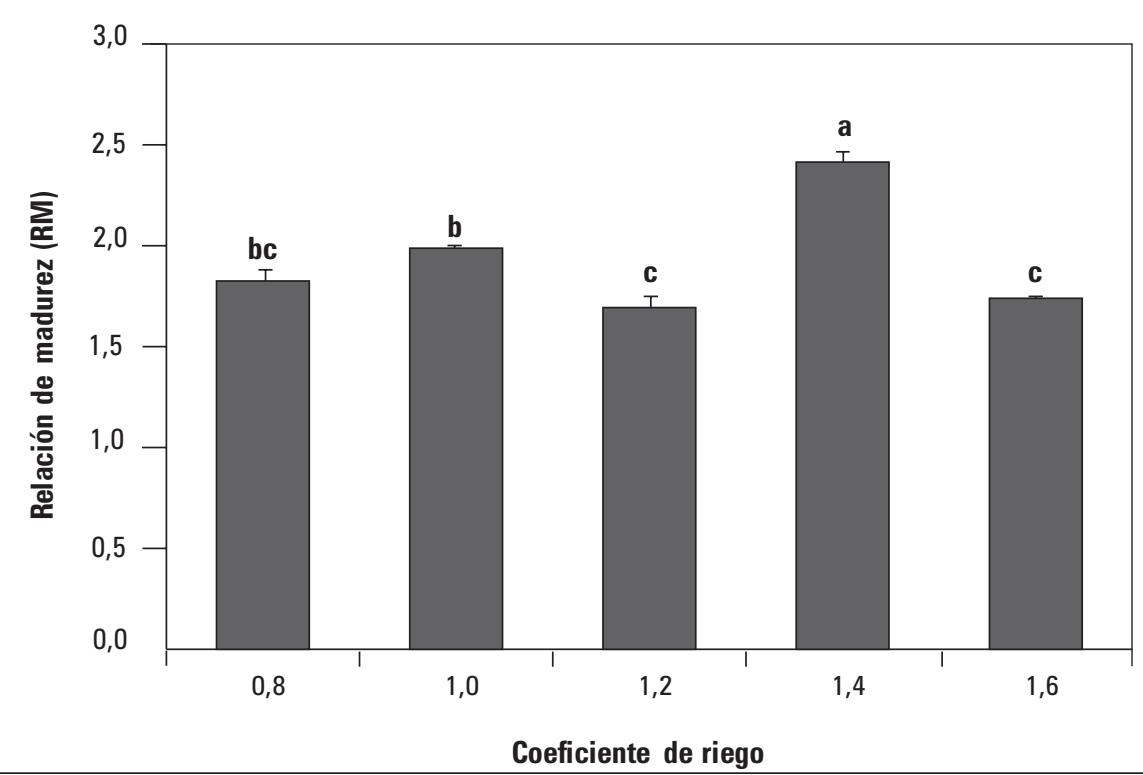

Figura 6. Relación de madurez (RM = SST/ATT) de frutos de fresa con aplicación de diferentes láminas de riego. Promedios con letras distintas indican diferencia significativa según la prueba de Tukey $(P \leq 0,05)$. Las barras de error indican el error estándar. 
go. El coeficiente de 0,8 tuvo la mayor eficiencia de agua con un 58\% (figura 7), no obstante, con esta lámina los rendimientos en cuanto a masa fresca de frutos fueron los más bajos. En el caso de los coeficientes de 1,2, 1,4 y 1,6 no se presentaron diferencias significativas con valores de $46,49 \%$, 42,51\% y $45,77 \%$, respectivamente. Lo anterior indica que mantener una mayor eficiencia en el uso del agua no siempre se ve reflejado en un mayor tamaño pero sí en los valores de SST y ATT. Lo cual es acorde a lo reportado por Savic et al. (2011) y Zotarelli et al. (2009), quienes afirman que cuanta menor agua se aplica a las plantas, mayor es la eficiencia en la producción de biomasa.

Las funciones de producción agua/rendimiento se han medido principalmente en cultivos anuales, y muy pocos frutales, donde se ha encontrado que al disminuir el agua en cultivos de vid a un $60 \%$ el rendimiento disminuye un $35 \%$ (Grimes y Williams, 1990). Erdem et al. (2005) encontraron resultados similares para el cultivo de sandía ya que obtuvieron funciones cuadráticas al relacionar la lámina de riego y el uso eficiente del agua de riego con el rendimiento de frutos. También Irmak et al. (2000) obtuvieron una función lineal positiva entre láminas de riego y el rendimiento de grano de maíz de 0,1 a $0,6 \mathrm{~kg} \mathrm{~m}^{-2}$, en un rango de 60 a $450 \mathrm{~mm}$.

La razón está en que el proceso base de la producción de la biomasa (la fotosíntesis) y el del gasto de agua (transpiración) se producen a la vez, y la entrada del $\mathrm{CO}_{2}$ y la salida del agua utilizan la misma vía a través de los estomas de las hojas. Cuanto más abiertos están, más fácilmente entra el $\mathrm{CO}_{2}$ pero también más rápidamente se escapa el agua (Medrano et al., 2007), con lo cual en situación de déficit hídrico, el balance favorece a la transpiración y a la baja acumulación de agua en los tejidos.

\section{CONCLUSIONES}

Aplicar una lámina de riego de 1,6 veces la evaporación promueve en las plantas de fresa una mayor acumulación de masa fresca de hojas,

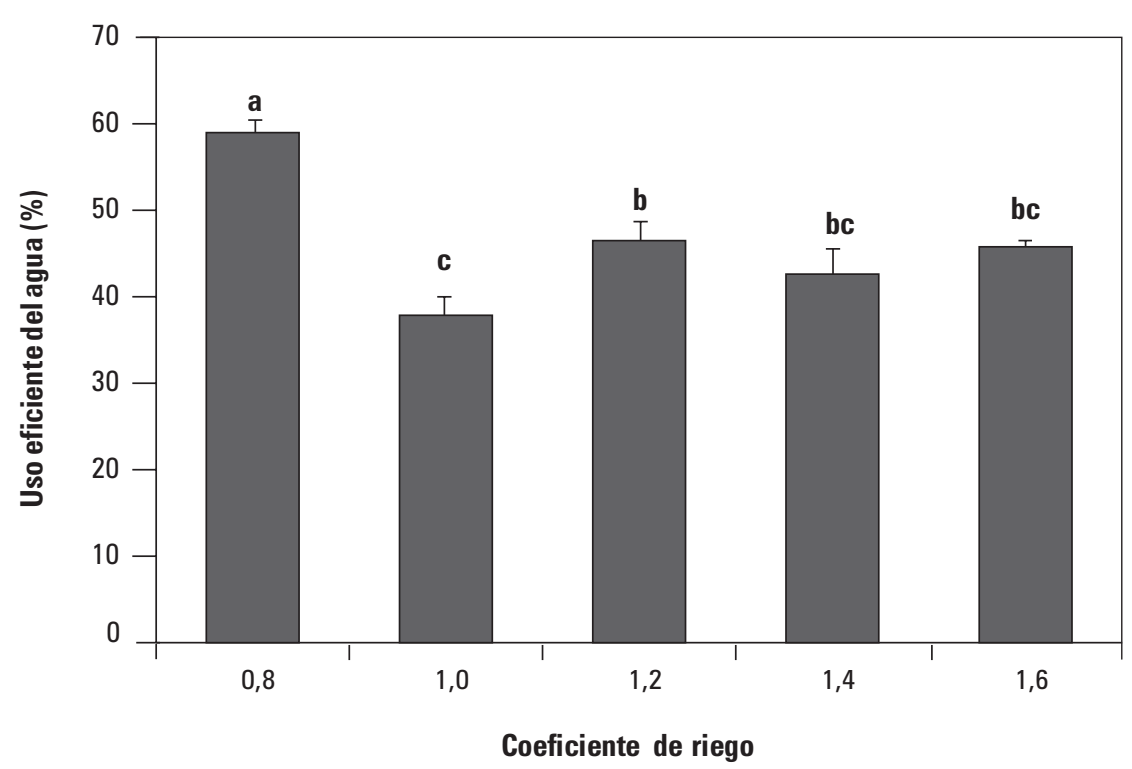

Figura 7. Uso eficiente del agua en plantas de fresa sometidas a aplicación de diferentes láminas de riego. Promedios con letras distintas indican diferencia significativa según la prueba de Tukey $(P \leq 0,05)$. Las barras de error indican el error estándar. 
masa seca de hojas y corona y peciolos, área foliar y SST, aun así, al disminuir la cantidad de agua aplicada a 1,4 veces la evaporación, la raíz de las planta de fresa se vio favorecida así como la masa seca de los frutos y la relación de madurez. La aplicación de una lámina de riego con un coeficiente multiplicador de la evaporación de 1,4 presenta el mayor rendimiento en cuanto a producción de frutos en las plantas de fresa, además esta aplicación mantiene una alta eficiencia en el uso del agua.

\section{AGRADECIMIENTOS}

Este trabajo formó parte del proyecto "Efecto de diferentes láminas y frecuencias de riego sobre la producción y calidad poscosecha de fresa (Fragaria sp.)" con código SGI 1068, realizado con el apoyo de Colciencias y la Universidad Pedagógica y Tecnológica de Colombia (UPTC), Tunja, mediante el programa nacional de formación de jóvenes investigadores e innovadores año 2012 "Virginia Gutiérrez de Pineda".

\section{REFERENCIAS BIBLIOGRÁFICAS}

Casierra-Posada, F., P. Sánchez, J. Castaño y R. Viasús. 2009. Identificación de la cantidad y frecuencia óptimas de riego para almácigos de café (Coffea arabica var. Castillo) en la subestación experimental de Cenicafé, Santander. Cienc. Agric. 7(2), 41-52.

Casierra-Posada, F. y Y.A. Vargas. 2007. Crecimiento y producción de fruta en cultivares de fresa (Fragaria sp.) afectados por encharcamiento. Rev. Colomb. Cienc. Hortic. 1(1), 21-32.

Cellier, F., G. Conejero, J.C. Breitler y F. Casse. 1998. Molecular and physiological responses to water deficit in drought-tolerant and drought-sensitive lines of sunflower. Plant Physiol. 116, 319-328. Doi: 10.1104/pp.116.1.319

Chaves, M.M., O. Zarrouk, R. Francisco, J.M. Costa, T. Santos, A.P. Regalado y M.L. Rodríguez. 2010. Grapevine under deficit irrigation: hints from physiological and molecular data. Ann. Bot. 105(5), 661676. Doi:10.1093/aob/mcq030

Cosgrove, W. y F. Rijisberman. 2014. World water vision: making wáter everybody's business. $2^{\text {a }}$ ed. FAO, Roma.

2008. Efecto del déficit de agua en el trasplante de plántulas de tomate (Solanum lycopersicum L.). Agron. Colomb. 26(2), 246-255.

Baracaldo, A., R. Carvajal, A.P. Romero, A.M. Prieto, F.J. García, G. Fischer y D. Miranda. 2014. El anegamiento afecta el crecimiento y producción de biomasa en tomate chonto (Solanum lycopersicum L.), cultivado bajo sombrío. Rev. Colomb. Cienc. Hortic. 8(1), 92-102.

Beckles, D. 2012. Factors affecting the postharvest soluble solids and sugar content of tomato (Solanum lycopersicum L.) fruit. Postharv. Biol. Tecnol. 63, 129-140. Doi:10.1016/j.postharvbio.2011.05.016
Dry, P.R., B.R. Loveys, M.G. Mccarthy y M. Stoll. 2001. Strategic irrigation management in Australian vineyards. J. Int. Sci. Vigne Vin. 35(1), 129-139.

Erdem, Y., A.T. Erdem, H. Orta y H. Okursoy. 2005. Irrigation scheduling for watermelon with crop water stress index (CWSI). J. Centr. Eur. Agric. 6(4), 449-460.

Favati, F., S. Lovelli, F. Galgano, V. Miccolis, T. Di Tommaso y V. Candido. 2009. Processing tomato quality as affected by irrigation scheduling. Sci. Hortic. 122, 562-571. Doi:10.1016/j.scienta.2009.06.026 
Galván-Tovar, M., J.K. Shibata, A. García-Esteva, P. Yañez, E. Martínez y L. Ruiz. 2003. Déficit hídrico en planta, acumulación de biomasa y área foliar en tres etapas vegetativas en frijol común. Agric. Técn. México 29(2), 101-111.

Global Water Partnership (GWP). 2000. Manejo integrado de los recursos hídricos. GWP, Estocolmo.

Grimes, D.W. y L.E. Williams. 1990. Irrigation effects on plant water relations and productivity of Thompson Seedless grapevines. Crop Sci. 30, 255 260. Doi: 10.2135/cropsci1990.0011183X00300002 $0003 x$

Hanson, B. y W. Bendixen. 2004. Drip irrigation evaluated in Santa Maria Valley strawberries. Calif. Agric. 58(1), 48-53. Doi: 10.3733/ca.v058n01p48

Heredia, J., J. Siller, M. Báez, E. Araiza, T. Portillo, R. García y M. Mury. 1997. Cambios en la calidad y el contenido de carbohidratos en frutas tropicales y subtropicales a nivel de Consejo Regulador de la Denominación de Origen Rioja. Bulletin de l'OIV-Organisation Internationale de la Vigne et du Vin 84(965), 347.

Icontec. 1996. Norma Técnica Colombiana NTC 4103. Fresa variedad Chandler. Especificaciones técnicas. Instituto Colombiano de Normas Técnicas y Certificación, Bogotá.

Irmak, S., D.Z. Hamman y R. Bastug. 2000. Determination of crop water stress index for irrigation timing and yield estimation of corn. Agron. J. 92, 1221-1227. Doi: 10.2134/agronj2000.9261221x

Jarma, A., C. Buitrago y S. Gutiérrez. 1999. Respuesta del crecimiento de la habichuela (Phaseolus vulgaris L. var. BlueLake) a tres niveles de radiación incidente. Rev. Comalfi 26(1-3), 62-73.

Kader, A. 2013. Postharvest technology of horticultural crops - An overview from farm to fork. Ethiop. J. Appl. Sci. Technol. 1, 1-8.

Kirnak, H., C. Kaya, D. Higgs, I. Bolat, M. Simsek y A. Ikinci. 2003. Effects of preharvest drip-irrigation scheduling on strawberry yield, quality and growth. Aust. J. Exp. Agric. 43, 105-111. Doi: 10.1071/EA02045

Krüger, E., G. Schmidt y U. Brückner. 1999. Scheduling strawberry irrigation based upon tensiometer measurement and a climatic water balance model. Sci. Hortic. 81, 409-424. Doi: 10.1016/S03044238(99)00030-8

Kumar, S. y P. Dey. 2011. Effects of different mulches and irrigation methods on root growth, nutrient uptake, water-use efficiency and yield of strawberry. Sci. Hortic. 127, 318-324. Doi: 10.1016/j.scienta.2010.10.023

León, M., G. Hernández y G. Sotomayor. 2003. Manejo de riego en fresa cultivada en organopónico. Rev. Cienc. Técn. Agropec. 12(1), 39-43.

Leskovar, G. y G. Piccinni. 2005. Yield and leaf quality of processing spinach under deficit irrigation. HortScience 40(6), 1868-1870.

Marouelli, W.A. y W.L. Silva. 2007. Water tension thresholds for processing tomatoes under drip irrigation in Central Brazil. Irrig. Sci. 25, 411-418. Doi: 10.1007/s00271-006-0056-6

Marschner, H. 2002. Mineral nutrition of higher plants. $2^{\text {a }}$ ed. Academic Press, London.

Medrano, H., J. Bota, J. Cifre, J. Flexas, M. Ribas y J. Gulías. 2007. Eficiencia en el uso del agua por las plantas. Invest. Geogr. 43, 63-84.

Merchán-Gaitán, J.B., R.L Ferrucho y J.G. Álvarez-Herrera. 2014. Efecto de dos cepas de Trichoderma en el control de Botrytis cinerea y la calidad del fruto en fresa (Fragaria sp.). Rev. Colomb. Cienc. Hortic. 8(1), 44-56.

Núñez, A., G. Hoogenboom y D. Nesmith. 2005. Drought stress and the distribution of vegetative and reproductive traits of a bean cultivar. Sci. Agric. 62(1), 18-22. Doi: 10.1590/S010390162005000100004

Ojeda-Real, L.A., R. Cárdenas-Navarro, P. Lobit, O. Grageda-Cabrera, E. Valencia-Cantero y L. Macías-Rodríguez. 2008. Efecto de la nutrición nítrica y sistemas de riego en el sabor de la fresa (Fragaria $x$ ananassa Duch.). Rev. Chapingo Ser. Hortic. 14(1), 61-70.

Osterloh, A., G. Ebert, W.H. Held, H. Schulz y E. Urban. 1996. Lagerung von Obst und Südfrüchten. Verlag Ulmer, Stuttgart.

Paliyath, G. y D. Murr. 2008. Biochemistry of fruits. pp. 19-50. En: Paliyath, G., D. Murr, A. Handa y S. Lurie (eds.). Postharvest biology and tecnology of fruits, vegetables and flowers. Wiley-Blackwell Publishing, Ames, IA.

Parés, J., A. Blanco y J. Val. 2010. Optimización en el uso del agua y del abonado nitrogenado en plantaciones de manzano (Malus $x$ domestica Borkh.) cultivadas en zonas vulnerables del valle Medio del Ebro: Influencia sobre la fisiología del crecimiento vegetativo, reproductivo y la calidad de fruto. Tesis de doctorado. Universidad de Zaragoza, Zaragoza, España. 
Patané, C., S. Tringali y O. Sortino. 2011. Effects of deficit irrigation on biomass, yield, water productivity and fruit quality of processing tomato under semiarid Mediterranean climate conditions. Sci. Hortic. 129, 590-596. Doi:10.1016/j.scienta.2011.04.030

Pires, R., M. Folegatti, F. Passos, F. Arruda y E. Sakai. 2006. Vegetative growth and yield of strawberry under irrigation and soil mulches for different cultivation environments. Sci. Agric. 63(5), 417-425. Doi: 10.1590/S0103-90162006000500001

Porro, D., M. Ramponi, T. Tomasi, L. Rolle y S. Poni. 2010. Nutritional implications of water stress in grapevine and modifications of mechanical properties of berries. Acta Hort. 868, 73-80.

Prieto, J.A., E. Lebon y H. Ojeda. 2010. Respuesta estomática de variedades de vid al estado hídrico del suelo y al déficit de presión de vapor de agua. Jornadas de Actualización en Riego y Fertirriego, 1113 agosto 2010, Mendoza, Argentina.

Ramírez-Palma, T., I. Hernández-López, R. Cruz-Ortega y S. Sánchez-Nieto. 2005. Efecto del déficit hídrico en la expresión de los transportadores de sacarosa en plantas de jitomate. pp. 1-6. En: Memorias del XIV Congreso de Bioenergética y Biomembranas, 13 a 18 noviembre de 2005, Oaxaca, México.

Rufat, J., A. Arbonés, P. Villar, X. Domingo, M. Pascual y J.M. Villar. 2010. Effects of irrigation and nitrogen fertilization on growth, yield and fruit quality parameters of peaches for processing. Acta Hort. 868, 87-94.

Ryu, D., M. Ryu, S. Chung, S. Hur, S. Hong, J.H. Sung y H. Kim. 2014. Variability of soil water content, temperature, and electrical conductivity in strawberry and tomato greenhouses in winter. J. Biosyst. Eng. 37, 39-46. Doi: 10.5307/JBE.2014.39.1.039

Satta, D., M.G. Mameli, M. Tedde, M. Satta y L. De Pau. 2011. Effects of different irrigation regimes on yield and berry composition of field grown myrtus communis L. in north-western Sardinia. Acta Hort. 889, 423-430.
Savic, S., R. Stikic, V. Zaric, B. Vucelic-Radovic, Z. Jovanovic, S. Djordjevic y D. Petkovic. 2011. Deficit irrigation technique for reducing water use of tomato under polytunnel conditions. J. Centr. Eur. Agric. 12(4), 597-607. Doi: 10.5513/JCEA01/12.4.960

Silber, A. y A. Bar-Tal. 2008. Nutrition of substratesgrown plants. pp. 291-339. En: Raviv, M. y J.H. Lieth (eds.). Soilless culture: Theory and practice. Elsevier, San Diego, CA.

Tadeo, F. 2000. Fisiología de las plantas y el estrés. pp. 481-498. En: Azcon-Bieto, J. y M. Talón (eds.). Fundamentos de fisiología vegetal. McGraw-Hill/ Interamericana de España, Barcelona, España.

Taiz, L. y E. Zeiger. 2006. Plant physiology. $4^{\text {th }}$ ed. Sinauer Associates Publishers, Sunderland, MA.

Urrestarazu, G.M. 2004. Tratado de cultivos sin suelo. Mundi-Prensa. $3^{\text {a }}$ ed. Madrid.

Vázquez-Gálvez, G., R. Cárdenas-Navarro y P. Lobit. 2008. Efecto del nitrógeno sobre el crecimiento y rendimiento de fresa regada por goteo y gravedad. Agric. Técn. México 34(2), 235-241.

Viloria, Z., A. Arteaga y R.L. Pire. 1998. Desarrollo radical del pimentón (Capsicum annuum L.) bajo tres distancias de siembra y su relación con el peso de los frutos. Bioagro 10(3), 80-83.

Wang, F., S. Kang, T. Du, F. Li y R. Oiu. 2011. Determination of comprehensive quality index for tomato and its response to different irrigation treatments. Agr. Water Manage. 98, 1228-1238. Doi: 10.1016/j. agwat.2011.03.004

Zeng, C., B. Zhi-Long y Y. Bao-Zhong. 2009. Determination of optimum irrigation water amount for drip-irrigated muskmelon (Cucumis melo L.) in plastic greenhouse. Agr. Water Manage. 96(1), 595-602. Doi:10.1016/j.agwat.2008.09.019

Zotarelli, L., J. Scholberg, M. Dukes, R. Muñoz-Carpena y J. Icerman. 2009. Tomato yield, biomass accumulation, root distribution and irrigation water use efficiency on a sandy soil, as affected by nitrogen rate and irrigation scheduling. Agr. Water Manage. 96, 23-34. Doi:10.1016/j.agwat.2008.06.007 